\title{
LOS PROCESOS DE IMPLEMENTACIÓN DE LOS PROGRAMAS DE MEJORA DEL APRENDIZAJE Y EL RENDIMIENTO (PMAR). PERSPECTIVAS DEL PROFESORADO, EL ALUMNADO Y LAS FAMILIAS
}

\section{PROCESSES OF IMPLEMENTATION OF THE PROGRAMS FOR THE IMPROVEMENT OF THE LEARNING AND THE PERFORMANCE (PMAR). PERSPECTIVES OF TEACHERS, STUDENTS AND FAMILIES}

\author{
Joan Amer Fernández ${ }^{1}$ \\ Andreu Mir Gual ${ }^{2}$ \\ Universitat Illes Balears, España
}

\section{RESUMEN}

Los Programas de Mejora del Aprendizaje y el Rendimiento (PMAR) son activados con la aprobación de la Ley Orgánica para la Mejora de la Cualidad Educativa (LOMCE) de 9 de Diciembre de 2013. Estos programas se enmarcan entre los programas de atención a la diversidad planteados desde la orientación educativa para combatir los procesos de desenganche, fracaso y abandono escolar. El artículo se propone como objetivo central analizar el incipiente proceso de implementación de los PMAR, a través de las valoraciones del alumnado, equipos docentes y familias. En concreto, se analiza el componente pedagógico del programa, el papel del profesorado, el perfil del alumnado y la relación del programa con sus trayectorias educativas. El enfoque metodológico es de carácter cualitativo y descriptivo, basado en entrevistas exploratorias. Con este objetivo, se han realizado entrevistas en tres centros educativos del municipio de Palma (Mallorca). Se han llevado a cabo un total de 14 entrevistas en las cuales han participado alumnado, docentes y familias. Los principales resultados apuntan una buena aceptación del programa, una inquietud por la reincorporación a la vía ordinaria (4ํㅡ을 tras participar en el

\footnotetext{
1 Correspondencia: Joan Amer Fernández: Dpto. Pedagogia i Didàctiques Específiques. Universitat Illes Balears. Cra.Valldemossa km 7.507122 Palma (Balears). Correo-e: joan.amer@uib.cat web: http://www.gifes.uib.es
} 
programa, un acento en la dimensión conductual y del rendimiento de los alumnos y unas trayectorias marcadas por las dificultades de aprendizaje.

Palabras clave: Desenganche escolar, fracaso escolar, abandono escolar, rendimiento académico, programas de atención a la diversidad.

\begin{abstract}
Programs for the improvement of the learning and the performance (PMAR, acronym after the name in Spanish) are activated with the pass of the Organic Law for the improvement of the Educational Quality (LOMCE) of 9th December 2013. From a view of educational orienteering, these programs are embedded within the programs for the attention to the diversity to overcome processes of school dropout and failure. This article analyses the recent process of implementation of PMAR, through the opinions of the students, teachers and families. Specifically, it is studied the pedagogical component of the program, the role of the teachers, the student profiles and the relationship of the program with their educational trajectories. The methodological approach is qualitative and descriptive, based on interviews. With this goal, interviews have been undertaken in three secondary schools of the municipality of Palma (Mallorca). 14 interviews have been done, to students, teachers and families. Main results highlight a good acceptance of the program, concern about the reincorporation to the regular way ( $4^{\text {th }}$ of Secondary Education) after participating in the program, a stress in the behavioural dimension and of the performance of the students, and trajectories defined by their difficulties for learning.
\end{abstract}

Key Words: school disengagement, school failure, school dropout, programs of attention to the diversity.

\title{
Introducción
}

El fenómeno del fracaso y el abandono escolar debe entenderse desde la perspectiva de proceso, por ello Fernández-Enguita, Mena y Riviére (2010) acuñan el término de desenganche escolar. Para hacer frente a los procesos de desenganche escolar, la legislación educativa española ha ido disponiendo diferentes programas de atención a la diversidad en la etapa de la enseñanza secundaria, desplegados desde los equipos de orientación pedagógica y entendidos como medidas para promover el éxito educativo de los jóvenes con desmotivación y desfase curricular (Vélaz de Medrano, 2005). Entre los ejemplos más recientes de este tipo de programas, encontramos los Programas de Mejora del Aprendizaje y el Rendimiento (PMAR). Atendiendo a la disposición legal, los PMAR son una propuesta ya incluida en la LOE, pero no activada en su momento y que se pone en marcha con la aprobación de la LOMCE (2013). Al tratarse de un programa de reciente implantación, es importante estudiar sus efectos y conocer las reacciones de los agentes implicados, para indagar sobre las eventuales nuevas dinámicas que suponga en el ámbito de los programas de atención a la diversidad.

Los PMAR se desarrollan a partir del segundo curso de la Educación Secundaria Obligatoria (ESO), a diferencia de los Programas de Diversificación Curricular (PDC) que incorporaban alumnado a partir del tercer curso de ESO. La finalidad de los PMAR, es utilizar una metodología específica a través de la organización de contenidos, actividades prácticas y ámbitos diferentes a los establecidos en el carácter general, para que el alumnado que participe en el programa, se pueda incorporar al cuarto curso de ESO por la vía ordinaria y, así, puedan titularse. No son 
programas a partir de los cuales, quién participa puede obtener el graduado de ESO, como ocurría con los de PDC.

El acceso a los PMAR se reserva a aquel alumnado que presenta dificultades relevantes de aprendizaje, no imputables a la falta de estudio o de esfuerzo. En este sentido, el equipo de orientación educativa podrá proponer la incorporación a dichos programas a las madres y a los padres o tutores legales de aquel alumnado que haya repetido algún curso en cualquiera de las etapas y se encuentren en el primer o segundo curso de ESO, sin estar en condiciones de promocionar.

En relación a la estructura, los centros tienen que organizar estos programas, con materias diferentes a las establecidas en el carácter general. En cada uno de los dos cursos, que forman parte de los PMAR, tienen que trabajarse cuatro ámbitos específicos: (1) el lingüístico y social (que incorpore las materias de geografía e historia), lengua castellana y literatura, y lengua catalana y literatura, (2) el científico y matemático (en el cual se tratan contenidos de matemáticas, física, química, bilogía y geología), (3) el de lenguas extranjeras (correspondiente a la materia de la primera lengua extranjera, normalmente inglés) y, finalmente, (4) el ámbito práctico (que suma contenidos de las materias de tecnología, educación plástica, visual y audiovisual). Además de los cuatro ámbitos, el horario del alumnado que participa en estos programas, se completa con dos horas de educación física y dos de tutoría.

Se crean grupos específicos para el alumnado que participa en estos programas. El planteamiento, persigue que los estudiantes, vayan asumiendo los objetivos de la etapa que cursan, además de la adquisición de las competencias necesarias para promocionar a cuarto curso de ESO. Se plantea una potenciación de la acción tutorial entendida como recurso educativo que pueda contribuir de manera especial a la mejora en el proceso de aprendizaje del alumnado. Cada grupo de PMAR tiene que contar con un tutor específico, a ser posible miembro del Departamento de Orientación Educativa, y, si las circunstancias lo permiten, que sea la misma persona durante todo el programa. Como norma general, los PMAR, plantean una agrupación formada entre 10 y 15 alumnos/as.

El programa debe ser elaborado por el profesorado miembro del Departamento de Orientación, en colaboración, con los distintos departamentos didácticos y coordinados por el jefe o la jefa de estudios. Una vez aprobado por el claustro, el PMAR, tiene que formar parte del Proyecto Educativo de Centro (PEC). Ya en vigor, el procedimiento para la incorporación del alumnado al programa, se inicia después de la segunda evaluación, cuando el equipo docente emite un informe en el cual, se indican los motivos por los cuáles se considera oportuno que, determinado alumnado, pase a formar parte del PMAR en el próximo curso. A partir de aquí, el Departamento de Orientación, tiene que realizar una valoración psicopedagógica de cada caso, en el cual tiene que concluir, con una evaluación sobre la conveniencia de incorporar a cada alumno o alumna al programa. En los casos que se considere oportuna la entrada de alumnado en el PMAR, el tutor o la tutora y el orientador o la orientadora, se reunirán con la familia para informarles de las características esenciales del programa y, plantear, la posibilidad de que su hijo o hija puedan participar. En esta reunión, es necesario registrar por escrito la opinión de la familia. En conjunto, se trata de un dispositivo, que quiere dar respuesta a las necesidades particulares de un grupo de alumnado que presenta dificultades curriculares. Estudios, como el que aquí se presenta, permite valorar la utilidad de una medida que se encuentra en fase de desarrollo.

El programa PMAR se enmarca en las estrategias de diversificación y flexibilización de itinerarios en la educación secundaria, dirigido a alumnado con trayectorias de desenganche y fracaso escolar y riesgo de abandono. Los potenciales procesos de inclusión (o marginalización) educativa que tienen lugar en las trayectorias de los alumnos en el sistema escolar ocupa el centro de la agenda de investigación de múltiples académicos internacionales (entre otros, Ainscow, 2005; Apple, 2008; Bernardi y Cebolla-Boado, 2014; Gazeley, 2010). Los programas 
PMAR deberían constituir una estrategia para la inclusión educativa, aunque son principalmente una medida paliativa y con un carácter potencialmente segregador, en la línea de lo que señalan Escudero, González y Martínez (2009) para los programas de atención a la diversidad. Según NESSE (2009), las personas en situación de fracaso o abandono escolar tienen mayor probabilidad de estar desempleadas o de acceder a trabajos precarios y poco remunerados. Además el fracaso y el abandono provocan costes sociales (baja cohesión) y económicos (menor productividad y menor recaudación de impuestos). Según la OCDE (2011), hay que adoptar tres perspectivas en el estudio del fracaso escolar. Desde una perspectiva sistémica, el fracaso escolar ocurre cuando el sistema educativo no consigue el objetivo de una educación inclusiva que fomente el aprendizaje de los estudiantes. Desde una perspectiva de las escuelas, el fracaso ocurre cuando la escuela no garantiza un entorno de aprendizaje adecuado. Desde una perspectiva individual, el fracaso escolar se puede definir como el fracaso del alumno en obtener un nivel mínimo de conocimiento y habilidades, que puede terminar conllevando el abandono (OCDE, 2011). En el presente artículo se adopta la segunda de las perspectivas, relativa a qué entorno de aprendizaje (en forma de programa de atención a la diversidad) proporciona la escuela. Por tanto, los programas DC se enmarcan en las políticas para garantizar una escuela inclusiva y combatir los fenómenos de fracaso y abandono escolar.

Los programas PMAR deben enmarcarse en las políticas de integración escolar llevado a cabo en Europa estas últimas décadas. Desde que en los años 60 aparecieran las primeras iniciativas en el norte de el continente, estos años se ha evidenciado una tendencia que apuesta por dar una respuesta a alumnado con dificultades. Según Prada (2014), el país "modelo" y pionero en cuanto a inclusión es Italia, que ya entre los años 70-90 apostó por la educación integradora. Dettori (2011) indica que el proceso que se plantea en Italia propone el paso de un primer enfoque clínico a un enfoque pedagógico y más social. Otro de los países pioneros en la materia es Finlandia que ha avanzado mucho en la educación basada en un modelo inclusivo. En Finlandia, las medidas específicas de apoyo a la diversidad están recogidas en todo el currículo educativo, desde la planificación, equipamiento, evaluación, asesoramiento y desarrollo de las necesidades educativas especiales. Asegurar los planes de rehabilitación, así como lo referente a la dotación de servicios relativos a la educación especial, es responsabilidad tanto de las Administraciones educativas y sociales como de los servicios de salud de cada autoridad local.

Atendiendo a los antecedentes del entorno, se observa una apuesta firme a favor de modelos de escuela inclusiva. En este punto, resulta interesante tener en cuenta que la educación inclusiva implica que todos los jóvenes y adultos de una determinada comunidad aprendan juntos al margen de su origen, sus condiciones personales, sociales o culturales, incluidos aquellos que presentan cualquier problema de aprendizaje. En este sentido, Blanco (2008) destaca que el foco de atención de la educación inclusiva es la transformación de los sistemas educativos y de las culturas, las prácticas educativas y la organización de las escuelas para que atiendan la diversidad de necesidades educativas del alumnado, y lograr el pleno aprendizaje y participación de cada niño. Se trata de una propuesta que no pone requisitos de entrada ni mecanismos de selección o discriminación de ningún tipo, para hacer realmente efectivos los derechos a la educación, a la igualdad de oportunidades y a la participación. En la escuela inclusiva todos los alumnos se benefician de una enseñanza adaptada a sus necesidades y no sólo los que presentan necesidades educativas especiales. En el presente artículo, será posible observar como se produce una desconexión entre el modelo inclusivo de educación y los PMAR que inciden más en planteamientos tendentes a separar el alumnado con problemáticas de aprendizaje del grupo de referencia.

El objetivo del artículo es investigar los factores eventuales de éxito del programa PMAR, a partir de su despliegue en los centros, observando, por un lado, cómo se lo apropian el profesorado y el centro, y, por otro lado, cómo lo vive el alumnado y las familias. Con este objetivo, nos interesa saber qué dice la literatura académica sobre: i) el diseño del dispositivo 
pedagógico del PMAR y de los programas de atención a la diversidad; ii) qué comentan sobre el papel del profesorado y el centro en el despliegue del programa; iii) qué comentan sobre el perfil del alumnado que participa en dichos programas; iv) qué dicen sobre su relación con las trayectorias y decisiones educativas de este alumnado.

Primero, a la hora de analizar el dispositivo pedagógico del PMAR, su reciente aparición implica la falta de estudios sobre el tema. Es importante dirigirnos a los programas de atención a la diversidad y, en concreto, a su antecedente, el Programa de Diversificación Curricular (PDC), del cual comparte buena parte de la filosofía, el planteamiento y las prácticas. Según Aramendi, Vega y Buján (2012), en los PDC se enseñaba al alumnado a resolver problemas y a buscar información. Para Navarro (2006), disponer de menos alumnado en clase en estos programas, permitía explicaciones más adaptadas y mayor comprensión. Luzón, Porto, Torres y Ritacco (2009) destacan la importancia del trabajo colaborativo y las metodologías contextualizadas. Valdelomar (2010) propone un aprendizaje activo, social, significativo y afectivo. Aprendizaje activo consistente en trabajar en proyectos. Aprendizaje social basado en trabajar en equipo y fomentar el debate. Aprendizaje significativo entendido como avanzar a partir de lo que sabe el alumnado de cada tema. $\mathrm{Y}$ aprendizaje afectivo que pone en el centro el tema emocional. Margiotta, Vitale y Santos (2014), también destacan la importancia de un aprendizaje activo, que vaya alienado con las necesidades del alumnado. En conjunto, Ainscow (2005), subraya que cabe priorizar los procesos de aprendizaje por encima de los dispositivos.

Segundo, para hablar sobre el papel del profesorado y del centro en el despliegue de los programas de atención a la diversidad, Tarabini, Curran, Montes y Parcerisa (2015) hacen referencia al concepto de "vinculación escolar". A partir de Fredricks, Blumenfeld y Paris (2004), exponen que el concepto incluye tres vertientes: la comportamental, ligada a la conducta del alumnado, en relación a aquello definido como estándar escolar; la emocional, vinculada al sentimiento de pertinencia y los lazos afectivos del alumnado con el profesorado, los compañeros y las compañeras y el centro; y la cognitiva, relacionada con la motivación para el aprendizaje (esfuerzo e inversión de tiempo). En concreto, para que los alumnos vinculen, Tarabini, Curran, Montes y Parcerisa (2015), explican que es clave el rol del profesorado en términos de ayuda y soporte. En esta línea, señalan Carvalho y Taveira (2014), los profesores influyen en los estudiantes, a través de la relación en la asignatura que enseñan y el asesoramiento y diálogo con los estudiantes respecto a cuestiones escolares, profesionales y personales. Por tanto, el profesorado, tiene un papel relevante en generar oportunidades de éxito escolar entre el alumnado. Los equipos de orientación educativa también juegan un papel para lograr una mejor "vinculación escolar", a través de una dedicación suficiente a los alumnos y una adecuada coordinación con los tutores, teniendo en cuenta la complejidad de las necesidades del alumnado (Cano, Mayoral, Liesa y Castelló, 2013).

Tercero, en referencia al perfil del alumnado que participa en los programas, cabe decir que la selección del alumnado se realiza por los equipos de orientación y el profesorado (Horcas, Bernad y Martínez, 2015). Por tanto, el centro tiene un papel central en la composición de los grupos. Estos autores, también señalan, que se trata de un alumnado que, a menudo, señala la desmotivación y la falta de utilidad de la experiencia escolar como motivos para su "desenganche" de la institución y la cultura escolar. A la vez, cuando el alumnado se define a sí mismo, hacen referencia a términos como la limitación, la incapacidad o la carencia (Horcas, Bernad y Martínez, 2015). Santana, García y Santana (2013) apuntan que también debe tenerse en cuenta el grado de madurez y la vocación poco definida de este tipo de alumnado, para poder incrementar ambas.

Cuarto, en cuanto a la relación del programa con las trayectorias y decisiones educativas del alumnado, debe hablarse de los factores familiares y de las expectativas y autovaloración de los estudiantes. Además de los factores asociados al contexto escolar, González, García, Ruiz y Muñoz (2015) afirman que la mayoría de estudios sobre fracaso/abandono escolar y trayectorias 
educativas también hacen referencia a factores asociados al contexto familiar (incluyendo aquí el contexto socioeconómico). Martín, Alemán, Marchena y Santana (2015) exponen que el abandono escolar estaría sobretodo asociado con familias de bajo nivel educativo, baja supervisión educativa, clima familiar conflictivo y situaciones de desocupación de la familia. En relación a la autovaloración de los estudiantes, Bonal et al. (2003), subrayan la influencia de la posición socioeconómica en la construcción de sus expectativas, que incluirían actitudes de conformismo y distanciamiento, así como bajas expectativas.

El objetivo general del artículo es identificar los factores eventuales de éxitos del PMAR. Los objetivos específicos son (1) analizar el componente pedagógico y el marco del programa; (2) estudiar el papel del profesorado y el contexto escolar; (3) conocer el perfil del alumnado que participa en estos programas; y (4) estudiar la relación del programa con sus trayectorias educativas. Los objetivos específicos disponen de sus dimensiones e indicadores:

Objetivo específico 1. Analizar el componente pedagógico y el marco del programa.

- Dimensión pedagógica/didáctica

- Indicador valoración general

- Indicador diferencias respecto vía ordinaria

- Indicador ventajas y utilidad PMAR

- Indicador valoración formato/diseño programa

- Dimensión contexto del programa y estrategias contra el abandono

- Indicador modelo atención diversidad del centro

- Indicador estrategias éxito educativo

Objetivo específico 2. Estudiar el papel del profesorado y del contexto escolar (vinculación escolar)

- Dimensión profesorado

- Indicador valoración profesorado y perfil buen profesor/a

- Dimensión centro

- Indicador despliegue del programa en el centro

Objetivo específico 3. Conocer el perfil del alumnado

- Dimensión descriptiva alumnado

- Indicador composición social

- Indicador perfil educativo

- Indicador procesos de selección del alumnado del programa

- Indicador expectativas y orientaciones del profesorado

Objetivo específico 4. Relación del programa con las trayectorias educativas

- Dimensión personal

- Indicador valoración como estudiante

- Indicador expectativas educativas

- Dimensión familiar

- Indicador rol familia en las expectativas y decisiones educativas 


\section{Método}

\section{Participantes}

La población de estudio incluye 4 tutores/as, 5 alumnos/as y 4 madres, para sumar un total de 13 entrevistas. Es importante señalar el hecho de disponer de tres tipos de actores diferentes (docentes, estudiantes y familias). También, cabe mencionar el valor añadido de contar con las versiones de las madres, más difíciles de reclutar en estudios de estas características. Los criterios de inclusión y exclusión de los participantes recogieron que fueran alumnos con distintos rendimientos académicos dentro del programa, así como una distribución equilibrada por género y etnia o procedencia poblacional. Las 13 entrevistas, se distribuyen en tres centros de titularidad concertada con estudios de educación secundaria, en el municipio de Palma (Mallorca). La selección de los centros corresponde a criterios de representatividad, que tienen en cuenta la composición sociológica y territorial de Palma. Así, se trata de tres escuelas, con alumnado socialmente heterogéneo, con presencia de clases medianas y trabajadoras, y población inmigrada. Los criterios de selección de los centros tuvieron en cuenta que un centro está en un barrio de clases trabajadoras, otro en un barrio de clase media y el tercero en un pueblo (clases media y baja). Los centros de titularidad concertada de estos barrios fueron los que presentaron accesibilidad y disponibilidad para participar en el estudio. A efectos de respetar la confidencialidad de las contribuciones de las entrevistas, los tres centros, serán codificados respectivamente como Palma Ponent, Palma Llevant y Palma Rural.

TABLA 1. Participantes

\begin{tabular}{lrrrrr}
\hline \multicolumn{7}{c}{ Centro Educativo } & & & \\
\hline Informante & Palma Ponent & Palma Rural & Palma Llevant & Total & $\%$ \\
\hline Alumno & 1 & 2 & 2 & 5 & $38.46 \%$ \\
Madre & 1 & 2 & 1 & 4 & $30.77 \%$ \\
Tutor & 1 & 1 & 2 & 4 & $30.77 \%$ \\
Total & 3 & 5 & 5 & 13 & $100.00 \%$ \\
$\%$ & $23.08 \%$ & $38.46 \%$ & $38.46 \%$ & $100.00 \%$ & $7.69 \%$ \\
\hline
\end{tabular}

Fuente: elaboración propia

\section{Instrumento}

Se ha utilizado la entrevista semiestructurada, con escucha activa y metódica (Bourdieu, 1999; Ballester, Nadal y Amer, 2014). Las preguntas están alineadas con los indicadores, las dimensiones y los objetivos de la búsqueda. En el guión de las entrevistas con el alumnado y los padres y las madres, los principales ítems, eran preguntas sobre la trayectoria educativa del alumnado, el instituto y el profesorado, el perfil del buen profesor/a, la valoración del programa PMAR, los cambios respecto a la vía ordinaria, la utilidad del PMAR, la valoración como estudiante, las expectativas educativas y los factores que influyen en las decisiones educativas de los jóvenes. En el guión de las entrevistas con el profesorado, los ítems preguntados, eran cuestiones sobre su trayectoria profesional, su situación en el centro, la composición social del alumnado, el perfil educativo, la selección del alumnado, las expectativas educativas, las orientaciones educativas al alumnado y los factores para el éxito educativo. 


\section{Procedimiento y análisis de las informaciones}

Se utiliza un diseño cualitativo con entrevistas a tres tipos de informantes (alumnos, tutores y madres), de cara a la triangulación metodológica. El guión de las entrevistas fue utilizado para una investigación previa sobre los Programa de Diversificación Curricular (Amer y Pascual, 2015). Se contacta los centros y se les explican los criterios de inclusión y exclusión de los participantes del estudio. Se procede a contactar a los participantes y realizar las entrevistas.

En cuanto al análisis de las informaciones, la propuesta metodológica utilizada es cualitativa, de análisis de contenido. Para el vaciado, se clasifica la información de las transcripciones de las entrevistas, mediante el programa NVIVO11 de análisis cualitativa de datos. La información recogida, a través de la entrevista semiestructurada, se categoriza a partir de los temas que aparecen en las respuestas de las personas protagonistas entrevistadas. Las preguntas del guión de la entrevista, estructuran las categorías previas, mientras que aquellas informaciones que van más allá de las preguntas planteadas al guión, se recogen como categorías emergentes.

En relación a la fiabilidad de las respuestas, se detalla el porcentaje de citas por categorías y dimensiones de análisis (Tablas $2 \mathrm{~A}$ y $2 \mathrm{~B}$ ), para conocer la densidad de las respuestas. En la gran mayoría de las dimensiones, la concordancia ha sido superior a un tercio (tanto en conjunto como para alumnos, madres y tutores por separado). Referente al índice de concordancia, no se puede informar sobre éste, porque no se ha confrontado la selección de diferentes opciones con los distintos participantes. La estrategia de recogida de la información se basaba en aportaciones libres.

TABLA 2A. Porcentaje de citas por categorías y dimensiones de análisis

\begin{tabular}{|c|c|c|c|c|c|}
\hline & \multicolumn{3}{|c|}{ Informante } & \multirow{2}{*}{ Total } & \multirow{2}{*}{$\%$} \\
\hline & Alumno & Madre & Tutor & & \\
\hline \multicolumn{6}{|c|}{ Objetivo 1. Analizar el componente pedagógico y el marco del programa } \\
\hline 1.1 Dimensión pedagógica-dispositivo PMAR & 4 & 3 & 3 & 10 & $66.66 \%$ \\
\hline Valoración general & 2 & 2 & 2 & 6 & $46.15 \%$ \\
\hline Diferencias vía ordinaria & 4 & 2 & 3 & 9 & $69.23 \%$ \\
\hline Ventajas PMAR & 4 & 2 & 0 & 6 & $46.15 \%$ \\
\hline Utilidad PMAR & 2 & 3 & 0 & 5 & $38.46 \%$ \\
\hline $\begin{array}{l}\text { 1.2 Dimensión contexto del programa y } \\
\text { estrategias }\end{array}$ & 0 & 0 & 4 & 4 & $30.77 \%$ \\
\hline Atención diversidad en el centro & 0 & 0 & 4 & 4 & $30.77 \%$ \\
\hline Estrategias éxito educativo & 0 & 0 & 3 & 3 & $23.08 \%$ \\
\hline \multicolumn{6}{|c|}{ Objetivo 2. Estudiar el papel del profesorado y del contexto escolar (vinculación escolar) } \\
\hline 2.1 Dimensión profesorado & 5 & 4 & 0 & 9 & $69.23 \%$ \\
\hline Valoración profesorado-perfil buen profesor & 5 & 4 & 0 & 9 & $69.23 \%$ \\
\hline 2.2 Dimensión centro & 0 & 0 & 2 & 2 & $15.38 \%$ \\
\hline Despliegue en el centro & 0 & 0 & 2 & 2 & $15.38 \%$ \\
\hline \multicolumn{6}{|l|}{ Objetivo 3. Conocer el perfil del alumnado } \\
\hline 3.1 Dimensión descriptiva alumnado & 0 & 0 & 4 & 4 & $30.77 \%$ \\
\hline Composición social & 0 & 0 & 2 & 2 & $15.38 \%$ \\
\hline Perfil educativo & 0 & 0 & 3 & 3 & $23.08 \%$ \\
\hline Selección alumnado & 0 & 0 & 4 & 4 & $30.77 \%$ \\
\hline Expectativas y orientaciones profesorado & 0 & 0 & 4 & 4 & $30.77 \%$ \\
\hline
\end{tabular}




\begin{tabular}{|c|c|c|c|c|c|}
\hline & \multicolumn{3}{|c|}{ Informante } & \multirow{2}{*}{ Total } & \multirow[b]{2}{*}{$\%$} \\
\hline & Alumno & Madre & Tutor & & \\
\hline \multicolumn{6}{|c|}{ Objetivo 4. Relación del programa con las trayectorias educativas } \\
\hline 4.1 Dimensión personal & 5 & 4 & 4 & 13 & $100.00 \%$ \\
\hline Valoración como estudiante & 4 & 4 & 0 & 8 & $61.54 \%$ \\
\hline Expectativas & 5 & 4 & 4 & 13 & $100.0 \%$ \\
\hline 4.2 Dimensión familiar & 5 & 4 & 0 & 9 & $69.23 \%$ \\
\hline $\begin{array}{l}\text { Indicador rol familia en las expectativas y } \\
\text { decisiones educativas }\end{array}$ & 5 & 4 & 0 & 9 & $69.23 \%$ \\
\hline
\end{tabular}

TABLA 2B. Porcentaje de citas por categorías y dimensiones de análisis, con detalle por grupos

\begin{tabular}{|c|c|c|c|c|c|c|c|c|c|}
\hline & \multicolumn{3}{|c|}{ Alumnos (5) } & \multicolumn{3}{|c|}{ Madres (4) } & \multicolumn{3}{|c|}{ Tutores (4) } \\
\hline & sí & no & \% sí & sí & no & \% sí & sí & no & \% sí \\
\hline \multicolumn{10}{|c|}{ Objetivo 1. Analizar el componente pedagógico y el marco del programa } \\
\hline 1.1 Dimensión pedagógica-dispositivo PMAR & 4 & 1 & $80.00 \%$ & 3 & 1 & $75.00 \%$ & 3 & 1 & $75.00 \%$ \\
\hline Valoración general & 2 & 3 & $40.00 \%$ & 2 & 2 & $50.00 \%$ & 2 & 2 & $50.00 \%$ \\
\hline Diferencias vía ordinaria & 4 & 1 & $80.00 \%$ & 2 & 2 & $50.00 \%$ & 3 & 1 & $75.00 \%$ \\
\hline Ventajas PMAR & 4 & 1 & $80.00 \%$ & 2 & 2 & $50.00 \%$ & 0 & 4 & $0.00 \%$ \\
\hline Utilidad PMAR & 2 & 3 & $40.00 \%$ & 3 & 1 & $75.00 \%$ & 0 & 4 & $0.00 \%$ \\
\hline 1.2 Dimensión contexto del programa y estrategias & 0 & 5 & $0.00 \%$ & 0 & 4 & $0.00 \%$ & 4 & 0 & $100.00 \%$ \\
\hline Atención diversidad en el centro & 0 & 5 & $0.00 \%$ & 0 & 4 & $0.00 \%$ & 4 & 0 & $100.00 \%$ \\
\hline Estrategias éxito educativo & 0 & 5 & $0.00 \%$ & 0 & 4 & $0.00 \%$ & 3 & 1 & $75.00 \%$ \\
\hline \multicolumn{10}{|c|}{ Objetivo 2. Estudiar el papel del profesorado y del contexto escolar (vinculación escolar) } \\
\hline 2.1 Dimensión profesorado & 5 & 0 & $100.00 \%$ & 4 & 0 & $100.00 \%$ & 0 & 4 & $0.00 \%$ \\
\hline Valoración profesorado-perfil buen profesor & 5 & 0 & $100.00 \%$ & 4 & 0 & $100.00 \%$ & 0 & 4 & $0.00 \%$ \\
\hline 2.2 Dimensión centro & 0 & 5 & $0.00 \%$ & 0 & 4 & $0.00 \%$ & 2 & 2 & $50.00 \%$ \\
\hline Despliegue en el centro & 0 & 5 & $0.00 \%$ & 0 & 4 & $0.00 \%$ & 2 & 2 & $50.00 \%$ \\
\hline \multicolumn{10}{|l|}{ Objetivo 3. Conocer el perfil del alumnado } \\
\hline 3.1 Dimensión descriptiva alumnado & 0 & 5 & $0.00 \%$ & 0 & 4 & $0.00 \%$ & 4 & 0 & $100.00 \%$ \\
\hline Composición social & 0 & 5 & $0.00 \%$ & 0 & 4 & $0.00 \%$ & 2 & 2 & $50.00 \%$ \\
\hline Perfil educativo & 0 & 5 & $0.00 \%$ & 0 & 4 & $0.00 \%$ & 3 & 1 & $75.00 \%$ \\
\hline Selección alumnado & 0 & 5 & $0.00 \%$ & 0 & 4 & $0.00 \%$ & 4 & 0 & $100.00 \%$ \\
\hline Expectativas y orientaciones profesorado & 0 & 5 & $0.00 \%$ & 0 & 4 & $0.00 \%$ & 4 & 0 & $100.00 \%$ \\
\hline \multicolumn{10}{|c|}{ Objetivo 4. Relación del programa con las trayectorias educativas } \\
\hline 4.1 Dimensión personal & 5 & 0 & $100.00 \%$ & 4 & 0 & $100.00 \%$ & 4 & 0 & $100.00 \%$ \\
\hline Valoración como estudiante & 4 & 1 & $80.00 \%$ & 4 & 0 & $100.00 \%$ & 0 & 4 & $0.00 \%$ \\
\hline Expectativas & 5 & 0 & $100.00 \%$ & 4 & 0 & $100.00 \%$ & 4 & 0 & $100.00 \%$ \\
\hline 4.2 Dimensión familiar & 5 & 0 & $100.00 \%$ & 4 & 0 & $100.00 \%$ & 0 & 4 & $0.00 \%$ \\
\hline $\begin{array}{l}\text { Indicador rol familia en las expectativas y decisiones } \\
\text { educativas }\end{array}$ & 5 & 0 & $100.00 \%$ & 4 & 0 & $100.00 \%$ & 0 & 4 & $0.00 \%$ \\
\hline
\end{tabular}

Fuente: elaboración propia 


\section{Resultados}

La exposición de los resultados de las entrevistas a tutores/as, alumnado y familias se ha alineado con los objetivos, las dimensiones y los indicadores de la búsqueda. Se recoge aquello aportado a las entrevistas referentes al componente pedagógico y el marco del programa, el papel del profesorado y el contexto escolar, el perfil del alumnado y la relación del programa con las trayectorias educativas. En la Tabla 3 incluimos el resumen de los resultados, por objetivos y dimensiones.

TABLA 3. Resumen de los resultados, por objetivos y dimensiones

\begin{tabular}{|c|c|c|c|}
\hline & Alumnos (5) & Madres (4) & Tutores (4) \\
\hline \multicolumn{4}{|c|}{ Objetivo 1. Analizar el componente pedagógico y el marco del programa } \\
\hline $\begin{array}{l}\text { 1.2 Dimensión contexto del } \\
\text { programa y estrategias }\end{array}$ & $\begin{array}{l}\text { - Grupo más pequeño y } \\
\text { mayor atención } \\
\text { profesorado. } \\
\text { - } \text { Desacuerdo con el hecho } \\
\text { que no exista en } 4 \text { ํSOSO } \\
\text { - Temario más adaptado y } \\
\text { reducido. } \\
\text { - Explicaciones más } \\
\text { adaptadas. }\end{array}$ & $\begin{array}{l}\text { - } \text { Nivel académico, más } \\
\text { adaptado al perfil } \\
\text { alumnado. } \\
\text { - Mayor atención } \\
\text { profesorado. } \\
\text { - Desacuerdo con el } \\
\text { hecho que no exista en } \\
4 \text { ํㅗㅇ. } \\
\text { - Mayor motivación de } \\
\text { los hijos. }\end{array}$ & 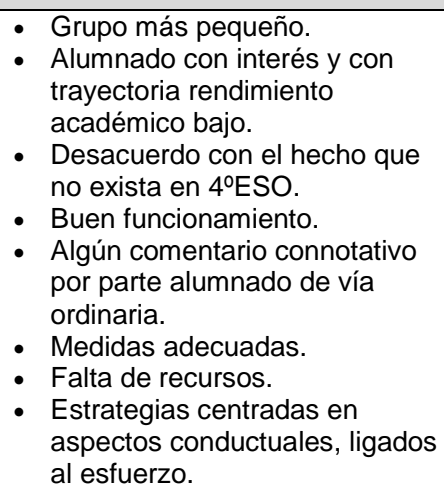 \\
\hline \multicolumn{4}{|c|}{ Objetivo 2. Estudiar el papel del profesorado y del contexto escolar (vinculación escolar) } \\
\hline 2.1 Dimensión profesorado & - Apoyo y comprensión & - Apoyo y comprensión & \\
\hline 2.2 Dimensión centro & & & $\begin{array}{l}\text { - Buen funcionamiento. } \\
\text { - } \quad \text { Clima positivo. } \\
\end{array}$ \\
\hline \multicolumn{4}{|c|}{ Objetivo 3. Conocer el perfil del alumnado } \\
\hline $\begin{array}{l}3.1 \text { Dimensión descriptiva } \\
\text { alumnado }\end{array}$ & & & $\begin{array}{l}\text { - Complejidad situaciones } \\
\text { personales y familiares. } \\
\text { - Nivel académico bajo. } \\
\text { - } \text { Falta de hábitos de estudio y } \\
\text { de aprendizaje. } \\
\text { - Relevancia aspectos } \\
\text { comportamentales. } \\
\text { - Motivación para titular. } \\
\text { - Vínculo emocional. }\end{array}$ \\
\hline \multicolumn{4}{|c|}{ Objetivo 4. Relación del programa con las trayectorias educativas } \\
\hline 4.1 Dimensión personal & $\begin{array}{l}\text { - } \text { Dificultades para el } \\
\text { estudio. } \\
\text { - } \text { Buen comportamiento. } \\
\text { - } \text { Vocación de trabajar para } \\
\text { aprobar. } \\
\text { - Expectativas educativas } \\
\text { alineadas con las } \\
\text { expectativas laborales en } \\
\text { trabajos de poca } \\
\text { cualificación. }\end{array}$ & $\begin{array}{l}\text { - Dificultades para el } \\
\text { estudio. } \\
\text { - Esfuerzo y conducta. } \\
\text { - Expectativas } \\
\text { educativas y laborales } \\
\text { similares a las de los } \\
\text { hijos (trabajo poco } \\
\text { cualificado). }\end{array}$ & $\begin{array}{l}\text { - Obtención del título de ESO. } \\
\text { - Orientación hacia ciclos } \\
\text { formativos. }\end{array}$ \\
\hline 4.2 Dimensión familiar & $\begin{array}{l}\text { - Importancia de las } \\
\text { familias, sobre todo de las } \\
\text { madres. } \\
\text { - Relevancia en la toma de } \\
\text { decisiones. }\end{array}$ & - Consejos y apoyo. & \\
\hline
\end{tabular}


En relación al primer objetivo, analizar el componente pedagógico del PMAR y el marco del programa, sobre la dimensión pedagógica y didáctica, se apuntan en las entrevistas aspectos sobre la valoración del programa y su diseño, sus beneficios y su utilidad, y las diferencias con la vía ordinaria.

En referencia a la valoración general y del diseño del programa, el alumnado entrevistado tiene una opinión positiva, destacando el grupo más pequeño y la mayor atención del profesorado. Las madres señalan el nivel académico, más adaptado al perfil del alumnado. En cuanto a los tutores y a las tutoras, destacan la dimensión reducida del grupo y señalan el interés del alumnado, siendo estudiantes con una trayectoria de rendimiento académico bajo. Tanto alumnado, como madres y tutores/as, tienen en común su desacuerdo al hecho que, en cuarto de ESO, el alumnado del programa tenga que volver a la vía ordinaria.

Me gustaría que en cuarto hubiera PMAR y te vuelves a pegar de morros. (Alumna 1 Palma Ponent)

En las entrevistas también se recogen las diferencias del programa con la vía ordinaria de ESO. El alumnado destaca que el temario está más adaptado y que es un poco más reducido. Las madres subrayan que el profesorado puede estar más por el alumnado. Los tutores y las tutoras apuntan que estos grupos, tienen muy buen funcionamiento, todo y que es cierto que a veces sienten, de manera puntual, algún comentario connotativo por parte de alumnado de la vía ordinaria.

Sobre las ventajas y la utilidad del programa, el alumnado destaca estar menos en el aula y que las explicaciones estén más adaptadas. Las familias subrayan la mayor motivación de sus hijos e hijas:

Mi hijo ha cambiado, se ha motivado y sigue adelante gracias a este Programa porque, si no, no seguiría de esta manera (...) Veo la idea súper buena. Yo destacaría la colaboración y yo sé que son menos alumnos y la verdad que en una clase normal, que hay el doble de alumnos, no es fácil de controlarlo pero aquí cada alumno es un mundo, cada alumno tiene su historia y vale la pena apoyar a cada uno individualmente. (Madre 1 Palma Rural).

En cuanto a la dimensión del contexto del programa y su encuadramiento en las estrategias contra el abandono escolar se recogen aspectos diversos. De los modelos de atención a la diversidad de los centros, los tutores y las tutoras señalan, la adecuación de las medidas pero, al mismo tiempo, la falta de recursos. En referencia a las estrategias para el éxito escolar (entendidas como aquellas estrategias que fomentan el aprovechamiento académico y la inclusión educativa), se apuntan aspectos conductuales, ligados al esfuerzo y mejorar la organización del alumnado, así como, la importancia de titular, como motivación.

Ligado al segundo objetivo, estudiar el papel del profesorado y el contexto escolar, sobre la dimensión de la valoración y el perfil del profesorado, tanto los estudiantes como las madres, señalan como buen profesor/a, a la persona que da apoyo y es comprensiva con el alumnado:

¿Qué es para ti un buen profesor? Pues cuando estás mal preguntarte qué te pasa o incluso si te cuesta estar ahí ayudando. (Alumna 2 Palma Llevant)

Un buen profesor es ese que te explica las veces que haga falta las cosas y que te apoye en todo momento en la asignatura. (Alumna 1 Palma Rural)

Pues que sepan entenderles, que les ayuden cuando lo necesiten, que si le cuesta le explique más. (Madre 1 Palma Ponent) 
Referente a la dimensión del centro educativo, ligada al despliegue del programa, se menciona el funcionamiento y el clima positivo. También, se comenta el cambio que supone pasar de ámbitos a asignaturas una vez empezado el curso, fruto de una indicación de la Consejería de Educación.

En relación al tercer objetivo, de conocer el perfil del alumnado, se recogen informaciones sobre el perfil social y educativo, los procesos de selección y las expectativas y las orientaciones del profesorado. Del perfil social y educativo, los tutores y las tutoras apuntan la complejidad de las situaciones personales y familiares del alumnado, así como, un nivel académico bajo y la falta de hábitos de estudio y de aprendizaje:

Es muy variado, pero básicamente son alumnos que no tienen una serie de habilidades diarias de trabajo, de estar en clase, enseguida se distraen, hay que cambiar constantemente de actividad para captar su atención. Luego hay alumnos que llevan un retraso bastante fuerte, alumnos que les cuesta mucho, problemas de hiperactividad, de déficit de atención, tenemos una alumna de otro país, que por ejemplo mi asignatura no la ha cursado nunca. En general hay muchos desfases. (Tutor 2 Palma Llevant)

En referencia a los procesos de selección, el profesorado comenta, la relevancia de los aspectos conductuales y la vocación de esfuerzo, conjuntamente, a un escenario de nivel académico bajo. En cuanto a las orientaciones y expectativas, ponen el acento, en la motivación y la vinculación emocional. También, son conscientes de las dificultades de cara a la obtención del título de ESO, que supone el retorno a $4^{\circ}$ de ESO, después de los dos años de PMAR. Por tanto, para la inclusión en el programa PMAR, el profesorado destaca el componente comportamental y la voluntad de trabajo.

Me gustaría, primero de todo, que en este curso que hacen, aprueben la mayoría, pero lo veo complicado, porque a medida de que vamos avanzando hay chicos que quedan atrasados por culpa suya, porque no estudian, lo que tienen que poner de su parte no lo hacen. Me gustaría que como mínimo estos alumnos que tengo más de la mitad aprobaran en junio y los otros entre junio y septiembre. De cara al año que viene, que pasaran a 4ํ de ESO normal, tengan más facilidad para sacarse el título de graduado escolar, lo veo bastante complicado, excepto 3, 4 ó 5 que lo han cogido en serio, los otros notarán un cambio bastante grande. (Tutor 1 Palma Llevant)

En cuanto al cuarto objetivo, la relación del programa con las trayectorias educativas, se tienen en cuenta la dimensión personal y la dimensión familiar. En la dimensión personal se recogen las autovaloraciones y las expectativas educativas. Las autovaloraciones recogen, entre otros, las dificultades para el estudio, el buen comportamiento y la vocación de trabajar para titular. Las madres también apuntan aspectos de conducta, de esfuerzo y de dificultad para el estudio. Veamos dos ejemplos de intervenciones del alumnado:

Me considero buena alumna pues porque, en primera, no quiero llevar ninguna suspendida, no la llevo y porque me quiero sacar el graduado. (Alumna 2 Palma Rural)

¿Te consideras un buen alumno? ¿Por qué? Sí, porque no paso de todo. Cuando tengo dudas pregunto, no me porto mal. (Alumna 1 Palma Ponent)

Las expectativas educativas del alumnado van muy alineadas con sus expectativas laborales. La mayoría de las personas entrevistadas, apuntan su voluntad, de trabajar en trabajos de poca cualificación (por ejemplo, dependientes o dependientas). Y en sus horizontes educativos se plantean titular y cursar un ciclo formativo de formación profesional. Las madres entrevistadas manifiestan posiciones bastante similares a las expresadas por los hijos y las hijas. Por último, los tutores y las tutoras, orientan hacia ciclos formativos $y$, a la vez, están preocupados y 
preocupadas por si, en primera instancia, el alumnado podrá obtener el título de graduado de ESO. En este sentido, las preocupaciones del profesorado recogidas en las entrevistas son principalmente de tipo académico y orientadas al logro escolar.

Creo que con esta adaptación sí que conseguiré la ESO (...) A mí me han aconsejado que me vaya a un FP de deporte por que se me da muy bien el deporte y me han dicho que me vaya allí, que puede que me lo saque

Entrevistadora: ¿De qué te gustaría trabajar de mayor? ¿Siempre has pensado lo mismo?

En una tienda de deporte, de botas o cosas así, pero si puedo sacarme una carrera de deporte mucho mejor. (Alumna 2 Palma Llevant)

Porque lo que se trabaja en el grupo a nivel de profesorado y yo como tutora y orientadora, se les intenta inculcar que ellos han de estudiar para poderse graduar y poder hacer luego una formación profesional, ya no sólo para tener una formación sino para conseguir un trabajo, que a ellos les guste, se sientan bien y, a su vez, puedan ganar un dinero para poder vivir. (Tutor 1 Palma Rural)

Por último, se analiza la dimensión familiar, prestando atención al rol de las familias en las expectativas y decisiones educativas. El alumnado entrevistado, coincide en señalar la importancia de sus familias, especialmente de las madres, en el momento de tomar decisiones.

Mi madre también me insiste que siga, ella me dice que tengo que seguir estudiando para ser alguien en la vida, ella no quiere que yo ande limpiando casas como hacen los demás, que siga mis estudios que me saque la ESO para poder ser alguien en la vida, poder tener un buen trabajo y todo esto. (Alumna 1 Palma Llevant)

Yo le digo que sí que tiene que avanzar porque ella puede. Porque yo veo a mi hija como trabaja en casa, sé la educación que tiene y los consejos que le damos los padres. (...) Con el apoyo de sus padres y de su hermana se ha hecho fuerte y ha dicho, "No, yo tengo que sacarme los estudios." (Madre 2 Palma Rural)

\section{Conclusiones}

Atendiendo a la estructura con la cual se han expuesto los resultados, el apartado de conclusiones establecerá un resumen de aquellos aspectos más substanciales de la reflexión, teniendo como punto de referencia los objetivos fijados previamente. Además, es importante recordar que el análisis de los PMAR se ha realizado a partir de las aportaciones de tres agentes diferentes: alumnado, profesorado y familias.

Por lo que hace referencia al primer objetivo, cabe decir que el análisis del componente pedagógico del programa ha mostrado una buena aceptación por parte de la mayoría de los entrevistados: se valora muy positivamente el hecho que sean grupos relativamente reducidos y que la atención que recibe el alumnado pueda ser más personalizada. Los mismos educadores y educadoras ponen de manifiesto que se trata de alumnado que cuenta con unos resultados académicos bajos en los cursos anteriores a su entrada en los PMAR. Aun así, los tutores y las tutoras aseguran que la mayoría consigue una mejora en su rendimiento, una vez han ingresado en el programa. Se constata que se trata de una medida de tipo paliativo, sin embargo, a diferencia de lo expresado en el marco teórico, en las entrevistas no se subraya el carácter 
potencialmente segregador (pese a trabajar en grupos separados) (Escudero, González y Martínez, 2009).

Con todo, existe una cierta preocupación, compartida por los tres agentes: si hasta ahora los Programas de Diversificación Curricular permitían que el alumnado pudiese titular, en el caso de los PMAR, no es así. De tal manera que, una vez cursados segundo y tercero de ESO, los integrantes del programa han de reincorporarse al cuarto ordinario. Este hecho genera una cierta incertidumbre, ya que hay dudas sobre la preparación que ofrece PMAR sea suficiente y adecuada para hacer frente al reto de aprobar la secundaria de manera ordinaria. Precisamente, esta última idea ha de relacionarse con el hecho que el alumnado entrevistado coincide en que observan menos temario que los demás y que lo realizan de una manera más adaptada.

Si nos sumergimos en la discusión del segundo objetivo (contexto escolar y papel del profesorado), conviene señalar algunas ideas substanciales. La continua falta de recursos es un argumento bastante recurrente en los discursos de los educadores y las educadoras de estos programas, los cuales también valoran aspectos conductuales ligados al esfuerzo y a la mejor organización del propio alumnado. En referencia al papel del profesorado, tanto los estudiantes como las madres, señalan como buen docente aquel que da apoyo y es comprensivo con el alumnado. En relación al contexto, en general, se destaca el adecuado funcionamiento del programa y el clima existente en las aulas. Además, también se comenta el cambio que supone pasar de ámbitos a asignaturas, una vez empezado el curso, todo como consecuencia de una determinación tomada por parte de la Administración.

En relación al tipo de alumnado, se recogen informaciones sobre el perfil social y educativo. Los educadores destacan la complejidad de las situaciones personales y familiares del alumnado, así como un nivel académico bajo y la falta de hábitos de estudio y de aprendizaje. En referencia a los procesos de selección, se destaca la dimensión conductual, el esfuerzo y los aspectos relacionados con el rendimiento académico. En este punto, se vuelven a poner de manifiesto los miedos de cara a la obtención del título de ESO, por el hecho de tener que incorporarse a un $4^{\circ}$ ordinario, después de haber cursado el PMAR.

El último de los objetivos que se marcan en el estudio tiene que ver con las trayectorias educativas. Dentro de ellas, se ha valorado la dimensión personal y ha sido, aquí, dónde se han identificado itinerarios vitales marcados por las dificultades en los procesos de aprendizaje, por el buen comportamiento y por la voluntad de trabajo a favor de un objetivo bastante nítido: obtener la titulación de ESO. En este punto, las familias, destacan las mejoras conductuales de algunos de sus hijos y sus hijas. Paralelamente a esta vertiente personal, también se han evaluado las expectativas educativas del alumnado, las cuales pertenecen ligadas a sus aspiraciones profesionales. La respuesta más compartida por el alumnado es la de aquellas personas que aspiran a trabajos de escasa cualificación. Los familiares mantienen unas expectativas muy parecidas. La obtención del título de ESO y de algún ciclo formativo de grado medio marcan el plan de ruta del alumnado entrevistado y, en esta voluntad, aparece la influencia directa de los mismos tutores y las mimas tutoras. Finalmente, el alumnado ha valorado muy positivamente el papel que juegan las familias en la tarea de apoyo y orientación en su propio proceso de toma de decisiones.

En relación al contraste de los resultados hallados con el marco teórico sobre el tema, hay que señalar la importancia del rol de los profesores en este tipo de programas, alineando la presente investigación con los planteamientos de Tarabini, Curran, Montes y Parcerisa (2015) relativos a la "vinculación escolar". En las entrevistas se apunta que, en los procesos de selección del alumnado por parte de los equipos de orientación educativa, son importantes los aspectos de la desmotivación y el bajo rendimiento académico. Estas razones también son subrayadas por Horcas, Bernad y Martínez (2015). En relación a las familias, en la línea de Bonal et al. (2003), las madres entrevistadas tienen bajas expectativas académicas y laborales de sus hijos. 
En referencia a las implicaciones prácticas del programa, se ha valorado el PMAR como un instrumento útil para poder inculcar determinados hábitos a este perfil de alumnado: pautas de organización de trabajo, rutinas de aprendizaje, etc. Además, el formato de estos grupos (no superan los 15 alumnos/as), permite poner en marcha - de una manera más ágil que no como en los grupos ordinarios - todo un conjunto de metodologías de enseñanza, con una mayor atención individualizada y más énfasis en las necesidades de los estudiantes, que pueden ser el punto de partida, de una mejora del rendimiento del alumnado que han estado expuestos y expuestas, anteriormente, a situaciones de fracaso escolar. En muchas ocasiones, se trata de personas que han acumulado frustraciones en sistemas más tradicionales dirigidos, fundamentalmente, a reforzar la parte cognitiva. Estos planteamientos del PMAR resultan ser más flexibles y, por ejemplo, permiten activar metodologías derivadas de la concepción de inteligencias múltiples (como ahora el trabajo cooperativo o por proyectos). Los planteamientos no sólo valoran la dimensión del conocimiento sino que también tienen en cuenta otras vertientes de la persona, como la competencia comunicativa, la creativa o la crítica. En este sentido, dada esta flexibilidad, algunos centros han hecho servir los cursos del PMAR para emprender proyectos innovadores que, en el caso que muestren unos resultados satisfactorios, se podrán extrapolar a otros cursos y niveles.

Pero sobre este campo de alternativas pedagógicas, en algunas ocasiones, se sitúa la rigidez de la norma. En este sentido, existen unas implicaciones políticas que, de alguna manera, condicionan la valoración que aquí se ha realizado de los PMAR. Existe una opinión bastante compartida que el programa abre todo un campo de dudas en relación a la titulación del final de la etapa. Docentes, familiares y alumnado identifican algunas dificultades cuando miran de analizar las posibilidades de reincorporación de un integrante del PMAR a un grupo ordinario para acabar la etapa de ESO, con las mismas condiciones, que los demás compañeros y compañeras. Existe un cierto consenso a la hora de pensar que la estructura de los antiguos PDC era más válida, dado que permitía la promoción del alumnado que participaba en el programa sin tener que volver al grupo ordinario para acabar 4ํㅜ de ESO. En esta misma dirección, el avance en el inicio de estos programas ha provocado que el proceso de selección y segregación del alumnado sea aún más primerizo que no como anteriormente. Todo junto, los distintos agentes consultados en la búsqueda que aquí se presenta, demuestran una voluntad explícita que los PMAR, puedan iniciarse en $3^{\circ}$ de ESO y no en $2^{\circ}$ de ESO, como fija la ley vigente.

Apuntando a las posibles limitaciones a las cuales ha tenido que hacer frente la investigación, se han de citar las dificultades para contactar con determinados colectivos, de tal manera que, no siempre ha sido sencillo el acceso a una muestra equilibrada. Un ejemplo de todo esto, lo tenemos, en la distribución de los participantes según el género: no se ha alcanzado una completa equidad entre las personas entrevistadas. Si bien las consultas con alumnado y tutores/as han sido, casi, inmediatas, no ha sido así con los familiares ya que, por cuestiones de trabajo u otros motivos, no siempre se han hecho presente en las entrevistas. Esta circunstancia ha terminado retrasando el proceso de recogida de información. Finalmente, el carácter incipiente de los programas PMAR, ha limitado el acceso a una bibliografía dónde aún no abunda. De hecho, las referencias a estos programas son escasas $y$, parece, que estos próximos años se irá alimentando el registro entorno a esta nueva realidad educativa. Además, tratándose de un estudio cualitativo, hay que tener en cuenta que las informaciones utilizadas para el análisis se centran en percepciones y valoraciones. Y, en el caso, del alumnado, en su propio autodiagnóstico.

En cuanto a las líneas de futuro, a partir del análisis que se ha expuesto, se abren algunas propuestas de cara a futuras investigaciones. Conjuntamente al estudio inicial, también, es de suma importancia realizar un seguimiento que permita establecer acciones de mejora. Los próximos cursos ya se dispondrá de datos relativos a la titulación de los participantes en el programa, la cual cosa se podrá complementar con estudios como ahora este, en el cual, se 
pueden recoger percepciones relativas a su puesta en marcha. Pero más allá de todo esto, es conveniente abrir otras vías de estudio, que permitan comparar esta alternativa con las demás opciones que se plantean para estos casos. Si bien los PMAR devienen una salida más bien académica para este perfil de alumnado tan singular, los Programas de Formación Profesional Básica (antiguos PCPI), representan una salida más vocacional. De esta manera, resultaría interesante, estudiar las diferencias substanciales de las dos propuestas y, si los criterios de selección del alumnado, que participa en estos programas, responden o no a la esencia que le da sentido a cada uno.

A modo de conclusión general, hay que alinear el programa PMAR con su antecedente el PDC. Con la salvedad del hecho que no existe el nuevo programa en el último año de la ESO, a partir de las entrevistas realizadas podemos determinar que la implementación y dinámicas de funcionamiento son similares: explicaciones más adaptadas y mayor comprensión en grupos reducidos, metodologías contextualizadas y aprendizaje social y significativo. Sin embargo, hay que enmarcar la puesta en funcionamiento del dispositivo PMAR en el contexto de la LOMCE (2013) que acentúa los procesos de aprendizaje basados en el rendimiento académico y sobre todo adelanta los procesos de segregación escolar (un curso académico antes).

\section{Agradecimientos}

Queremos agradecer a los centros educativos y a las personas entrevistadas su disponibilidad y facilidades para participar en el estudio. También, queremos dejar constancia de nuestro agradecimiento a María Félix y Lidia Gili, por su labor de trabajo de campo y transcripción de las entrevistas.

\section{Referencias bibliográficas}

Ainscow, M. (2005). Developing inclusive education systems: what are the levers for change? Journal of Educational Change, 6, 109-124

Amer, J. y Pascual, B. (2015). Las perspectivas del profesorado y el alumnado sobre la implementación de los programas de diversificación curricular en institutos de enseñanza secundaria del municipio de Palma (Mallorca). Profesorado, 19 (3), 126-138.

Apple, M. (2008). Can schooling contribute to a more just society? Education, Citizenship and Social Justice, 3 (3), 239-261.

Aramendi, P., Vega, A. y Buján, K. (2012). Los programas de diversificación curricular y los programas de cualificación profesional inicial ¿una alternativa al fracaso escolar? Revista Española de Pedagogía, 252, 237-256.

Ballester, L., Nadal, A. y Amer, J. (2014). Métodos y técnicas de investigación educativa. Palma: Universitat de les Illes Balears.

Bernardi, F. y Cebolla-Boado, H. (2014). Previous School Results and Social Background: Compensation and Imperfect Information in Educational Transitions. European Sociological Review, 30 (2), 207-217.

Blanco, R. (2008). Construyendo las bases de la inclusión y la calidad de la educación en la primera infancia. Revista de Educación, 347, 33-54. 
Bonal, X. (Dir.), Alegre, M.A., González, I., Herrera, D., Robira, M. y Saurí, E. (2003). Apropiacions escolars. Usos i sentits de l'educació obligatòria en l'adolescència. Barcelona: Octaedro.

Bourdieu, P. (1999). La miseria del mundo. Madrid: Akal.

Cano, M., Mayoral, P., Liesa, E. y Castelló, M. (2013). Valoración de las funciones del profesor de orientación educativa en Cataluña. Revista Española de Orientación y Psicopedagogía, 24 (3), 80-97.

Carvalho, M. y Taveira, M. (2014). El papel del profesorado en la toma de decisiones académicas y profesionales del alumnado. Revista Española de Orientación y Psicopedagogía, 25, 3, 2035.

Dettori, F. (2011). La integración de alumnos con necesidades educativas en Europa: el caso de España e Italia. Revista Educación Inclusiva, 4 (3), 67-77.

Escudero, J. M., González, M. T., \& Martínez, B. (2009). El fracaso escolar como exclusión educativa: comprensión, políticas y prácticas. Revista Iberoamericana de Educación, 50, 4164.

Fernández-Enguita, M., Mena, L. y Riviére, J. (2010). Fracaso y abandono escolar en España. Barcelona: La Caixa.

Fredricks, J.A, Blumenfeld, P.C., y Paris, A.H. (2004). School Engagement: Potential of the Concept, State of the Evidence. Review of Educational Research, 74(1), 59-109.

Gazeley, L. (2010). The role of school exclusión process in the re-production of social and educational disadvantage. British Journal of Educational Studies, 58, 3, 835-853.

González, S., García, P., Ruiz, F, y Muñoz, J.M. (2015). Factores de riesgo del abandono escolar desde la perspectiva del profesorado de educación secundaria obligatòria en Andalucía (España). Profesorado, 19 (3), 226-245.

Horcas, V., Bernad, J.C. y Martínez, I. (2015) ¿Sueña la juventud vulnerable con trabajos precarios? La toma de decisiones en los itinerarios de (in/ex)clusión educativa. Profesorado. Revista de currículum y formación del profesorado, 19 (3), 210-225.

Luzón, A., Porto, M., Torres, M., y Ritacco, M. (2009). Buenas prácticas en los programas extraordinarios de atención a la diversidad en centros de educación secundaria. Una mirada desde la experiencia. Profesorado. Revista de currículum y formación del profesorado, 13(3), 217-238.

Margiotta, U., Vitale, G., y Santos, J. S. (2014). The early school leaving phenomenon in the new millennium Europe: data, policies, interventions and perspectives. Cadernos CEDES, 34 (94), 349-366.

Martín, J.C., Alemán, J., Marchena, R. y Santana, R. (2015). El contexto familiar del alumnado en riesgo de abandono escolar temprano según la tipología familiar. Profesorado, 19 (3), 246253.

Navarro, R. (2006). Los Programas de Diversificación Curricular: ¿qué opinan los alumnos que los han cursado? Revista Española de Pedagogía, 233, 123-142.

NESSE, Network of Experts in Social Sciences of Education and Training (2009). Early school leaving. Lessons from research for policy makers, Recuperado de: http://www.nesse.fr/nesse/activities/reports

OCDE (2011). Equity and quality in education: supporting disadvantaged students and schools. París: OCDE.

Prada, D. (2014). Evolución del concepto de atención a la diversidad. Valladolid: Universidad de Valladolid. 
Santana, L., García, L., y Santana, J. (2013). Madurez y autoeficacia vocacional en $3^{\circ}$ y $4^{\circ}$ de ESO, Bachillerato y ciclos formativos. Revista Española de Orientación y Psicopedagogía, 24 (3), 8-26.

Tarabini, A., Curran, M., Montes, A. y Parcerisa, L. (2015). La vinculación escolar como antídoto del abandono escolar prematuro: explorando el papel del habitus institucional. Profesorado, 19 (3), 196-213.

Valdelomar, I. (2010). El profesorado de diversificación curricular. Orientaciones sobre formas de trabajo. Recuperado de: http://creena.educacion.navarra.es

Vélaz de Medrano, C. (2005). Medidas para promover el éxito educativo y sociolaboral de los jóvenes con desmotivación y desfase curricular en la ESO. Kikiriki. Cooperación Educativa, 78 (5).ALCUDIA, R y COL. (2000). Atención a la diversidad. Barcelona: Graó.

Fecha de entrada: 18 Agosto 2016

Fecha de revisión: 10 Noviembre 2016

Fecha de aceptación: 22 Noviembre 2016 